\title{
The Role of the Level of Interleukin-33 in the Therapeutic Outcomes of Immunotherapy in Patients with Allergic Rhinitis
}

\author{
Wail Fayez Nasr ${ }^{1}$ Samir Sorour Sorour ${ }^{1}$ Atef Taha El Bahrawy ${ }^{1}$ Ghada Samir Boghdadi ${ }^{2}$ \\ Alia A El Shahaway ${ }^{2}$ \\ ${ }^{1}$ Department of Otorhinolaryngology and Head and Neck Surgery, \\ Address for correspondence Wail Fayez Nasr, MD, Department of \\ Zagazig University Faculty of Human Medicine, Zagazig, \\ El Sharkeya, Egypt \\ 2 Department of Microbiology and Immunology, Zagazig University \\ Faculty of Human Medicine, Zagazig, El Sharkeya, Egypt \\ Otorhinolaryngology and Head and Neck Surgery, Zagazig University \\ Faculty of Human Medicine, 12 Abo Bakr Esedeek Villat El Gamma \\ Zagazig, Zagazig, El Sharkeya 4415, Egypt \\ (e-mail: wail.fayez@yahoo.com).
}

Int Arch Otorhinolaryngol 2018;22:152-156.

\begin{abstract}
Introduction Allergic rhinitis (AR) affects up to $40 \%$ of the population and results in nasal itching, congestion, sneezing, and clear rhinorrhea.

Objectives This study aimed to evaluate the changes in the clinical symptoms and in the level of serum interleukin (IL)-33 before and after pollen immunotherapy (IT) in patients with AR.

Methods The total symptom score and the levels of total immunoglobulin $\mathrm{E}(\mathrm{IgE})$ and IL-33 were determined in the serum of 10 non-allergic healthy controls and 45 patients with AR who were equally divided into 3 groups: GI (patients did not receive IT), GII (patients had received IT for 6 months) and GIII (patients had received IT for 2 years).

\section{Keywords}

- immunotherapy

- allergic rhinitis

- helper T cells

- allergen immunotherapy

Results There was a significantly higher concentration of IgE and IL-33 in the serum of patients with AR than in that of non-allergic patients. Furthermore, serum level of IL-33 decreased significantly after pollen IT. But, there was no significant reduction in the serum level of IL-33 between GIl and GIII patients.

Conclusion Our results show a clinical improvement associated with a decrease in serum level of IL-33 after pollen IT.
\end{abstract}

\section{Introduction}

Allergic rhinitis (AR) affects up to $40 \%$ of the population and results in nasal itching, congestion, sneezing, and clear rhinorrhea. Allergic rhinitis causes extranasal untoward effects, including reduced quality of life, declined sleep quality, and obstructive sleep apnea. ${ }^{1}$

Numerous controlled clinical trials have revealed the efficacy of specific allergen immunotherapy (SIT) in decreasing the clinical symptoms and costs associated with AR. Compared with pharmacotherapy, SIT may afford persistent clinical benefits after therapy cessation. Subcutaneous and sublingual immunotherapy are the two most extensively prescribed SIT routes worldwide. ${ }^{2}$

Interleukin (IL)-33, which is a member of the IL-1 family of cytokines, is now recognized as an important contributor to $\mathrm{T}$ helper 2 (Th2)-type immune responses. It was found that IL33 was elevated in the nasal secretions of AR patients. Furthermore, IL-33 in nasal secretions correlated significantly with the total nasal symptom score. ${ }^{3}$ Another study reported raised level of IL-33 in the sera of patients with AR who were allergic to tree and/or grass pollen. The association of IL-33 with the disease severity proposes that IL-33 is involved in the pathogenesis of intermittent AR. ${ }^{4}$ The current received

October 19, 2016

accepted

July 3, 2017

published online

August 28, 2017
DOI https://doi.org/

10.1055/s-0037-1605596. ISSN $1809-9777$.
Copyright $\odot 2018$ by Thieme Revinter

Publicações Ltda, Rio de Janeiro, Brazil
License terms

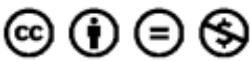


work was undertaken to analyze the role of immunotherapy in the treatment of AR and to follow the IL-33 levels in the serum of AR patients during the course of subcutaneous immunotherapy.

\section{Methods}

\section{Subjects}

This study was performed in the departments of otorhinolaryngology and microbiology and immunology of the Faculty of Medicine, Zagazig, Egypt, between May and August of 2015. Ten non-allergic healthy volunteers (ages 18-42 years, 4 females and 6 males), and 45 patients (ages 18-36 years, 25 females and 20 males) with AR date palm pollen were equally divided into three groups. Group I (GI) - patients did not receive immunotherapy, Group II (GII) - patients had initiated IT 6 months before starting the study, and Group III (GIII) patients had initiated IT 2 years before starting the study. The ethical committee of the hospital approved the study and written informed consent was obtained from each individual. A detailed clinical history and a complete physical examination were performed for each patient. Each subject in the nonallergic group was selected according to the following criteria: no history of allergic disease or nasal diseases, no pregnancy or lactation, and negative skin prick test. Each subject in the allergic group was selected randomly from AR patients attending the allergy outpatient clinic based on the following criteria: history of persistent rhinitis for at least 2 years, positive skin prick test to Phoenix dactylifera pollen only (5-mm wheal) and no evidence of treatment with IT during the previous 10 years. Exclusion criteria included (1) acute or chronic infectious or inflammatory diseases (asthma, atopic dermatitis), (2) anatomical abnormalities of upper respiratory tract, (3) those undergoing chronic treatment with systemic steroids or with systemic immunological disorders.

\section{Nasal Symptom Scores}

The atopic patients recorded the nasal symptoms scores 1 week before IT and again at 6 months and then at 2 years after treatment. The severity of each individual nasal symptom score (INSS), including nasal rhinorrhea, sneezing, itching, and congestion, was assessed on a scale of 0 to 3 ( $0=$ no symptom, 1 = mild symptom, 2 = moderate symptoms, and or 3 = severe symptoms). The total nasal symptom score (TNSS) was the sum of the scores for the individual symptoms. Total nasal symptom score values $(0-12)$ were categorized as mild (0-4), moderate (5-8), or severe $(9-12) .^{5}$

\section{Skin Prick Test (SPT)}

Skin prick test was performed by the same experienced personnel at the volar site of each forearm. they have applied one drop of each allergen extract (Allergy Laboratories Inc., Oklahoma City, USA) from a panel containing: house dust mite (Dermatophagoides pteronyssinus), aspergillus species mix, cottonwood mix, ash mix, tobacco leaf, rye-grass, and phoenix dactylifera pollen to the skin; at least $3 \mathrm{~cm}$ apart, according to a previously validated protocol. ${ }^{6}$ Histamine was used as a positive control and diluents of each allergen
(Allergy Laboratories Inc., Oklahoma City, USA) as a negative control The sensitivity of the skin test was determined by the size of the wheel. The largest diameter of the wheal was assessed as the size of the wheal after 20 minutes. A wheal diameter $3 \mathrm{~mm}$ or greater, accompanied by erythema, was considered as a positive reaction.

\section{Mode of Subcutaneous IT}

The treatment followed the manufacturers' instructions. The patients were received subcutaneous injections of standardized Phoenix dactylifera pollen extracts. Injections were received twice weekly up to a maintenance dose, and thereafter injections were received twice monthly. The maximum tolerated dose of $0.1 \%$ to $5 \%$ Phoenix dactylifera pollen extract preparation was achieved in 9 months. After each injection, the patients were instructed to remain under our supervision for a minimum of 30 minutes and to state any symptoms they may have experienced. Only oral antihistamines and mid-potency topical steroids were administrated concomitantly with the IT during the pollen season. However, these drugs withdrawn at least 4 weeks before collection of blood samples.

\section{Measurement of Total IgE and IL-33}

Blood samples were obtained from the patients who did not receive IT, the patients who received IT for 6 months and for 2 years and from the healthy controls were left at room temperature for 30 minutes until they coagulated. They were then centrifuged at 2,000 rpm for 10 minutes until serums were obtained. The serum samples were stored at $-70 \mathrm{C}$ until the study. The total IgE and IL-33 were determined in the serum samples. The total IgE levels were measured by enzyme linked immunosorbent assay (ELISA) according to the Ridascreen A0141 kit manufacturer's instructions (R-Biopharm AG, Darmstadt, Germany). Interleukin-33 was determined by ELISA according to the kit manufacturer's instructions, LEGEND MAX Human IL-33 ELISA Kit with pre-coated plates (Biolegend, San Diego, USA). The minimal detection level of IL-33 was $4.14 \mathrm{pg} / \mathrm{mL}$.

\section{Statistical Analysis}

Continuous variables are expressed as means ( \pm SD) and categorical values as percentages. We used the chi-square test for comparison of qualitative data and the analysis of variance (ANOVA) test for comparison between different groups. A $p$ value lower than 0.05 was considered statistically significant. The correlation between different biomarkers in AR patients was assessed using Pearson correlation test. Analysis was performed using the IBM SPSS software (SPSS Inc, Chicago, Il).

\section{Results}

Forty-five patients with allergic rhinitis to date palm pollen and ten non-atopic healthy volunteers enrolled in the study as a control. The demographic and clinical characteristics of study subjects are shown in - Table 1. Significant improvement of 
Table 1 The demographics, clinical and laboratory characteristics of the study subjects

\begin{tabular}{|l|l|l|l|l|}
\hline & $\begin{array}{l}\text { GI } \\
(\boldsymbol{n}=\mathbf{1 5})\end{array}$ & $\begin{array}{l}\text { GII } \\
(\boldsymbol{n}=\mathbf{1 5})\end{array}$ & $\begin{array}{l}\text { GIII } \\
(\boldsymbol{n}=\mathbf{1 5})\end{array}$ & $\begin{array}{l}\text { Control } \\
(\boldsymbol{n}=\mathbf{1 0})\end{array}$ \\
\hline Gender (male/female) & $7 / 8$ & $7 / 8$ & $6 / 9$ & $6 / 4$ \\
\hline Age (years) & $24.9 \pm 7.3$ & $25.5 \pm 7.2$ & $24.3 \pm 4.5$ & $24.9 \pm 8.6$ \\
\hline Duration of IT treatment & - & 6 months & 2 years & - \\
\hline Nasal rhinorrhea & 8 & 6 & 3 & - \\
\hline Itching & 13 & $3^{* *}$ & $0^{* *}$ & - \\
\hline Sneezing & 15 & $5^{* *}$ & $5^{* *}$ & - \\
\hline Obstruction & 14 & $2^{* *}$ & $0^{* *}$ & - \\
\hline Total IgE IU/mL & $216.3 \pm 80.8^{*}$ & $233.3 \pm 67.9^{*}$ & $188.6 \pm 29.7^{*}$ & $35.2 \pm 34.6$ \\
\hline IL-33 pg/mL & $117.7 \pm 64.7$ & $30.04 \pm 5.42^{* *}$ & $13.9 \pm 3.14^{* *}$ & $17 \pm 2.9$ \\
\hline
\end{tabular}

Abbreviation: IgE, immunoglobulin E; IL, interleukin; IT, immunotherapy; $n$, number of subjects.

* Significant differences from control group, ${ }^{* *}$ Significant differences from GI.

sneezing, itching and obstruction was observed in GII and GIII AR patients when compared with GI AR patients $(p<0.0001)$ (-Fig. 1). Increase in the duration of IT was accompanied by clinical improvement in symptoms.

\section{Serum Levels of Total IgE and IL-33}

The results are shown in - Table 1. The serum levels of total IgE in patients with AR (GI, GII and GIII) were statistically highly significant compared with those in the control group $(p<0.001)$. No significant differences were observed in the total IgE levels among the groups of AR patients. Serum levels of IL-33 were found significantly higher in the GI AR patients $(117.7 \pm 64.7 \mathrm{pg} / \mathrm{mL})$ than in the control group $(17 \pm 2.9)$ $(p<0.001)$. Serum levels of IL-33 were significantly lower in GII and GIII AR patients $(30.04 \pm 5.42 \mathrm{pg} / \mathrm{mL}, 13.9 \pm 3.14 \mathrm{pg} /$ $\mathrm{mL}$ respectively) than in GI AR patients $(117.7 \pm 64.7 \mathrm{pg} / \mathrm{mL})$ $(p<0.001)$ (-Fig. 2). However, no statistically significant differences were present between GII and GIII AR patients despite the trend toward lower IL-33 levels in GIII AR patients. There was no significant correlation between IL33 levels and total IgE in GI, GII and GIII AR patients.

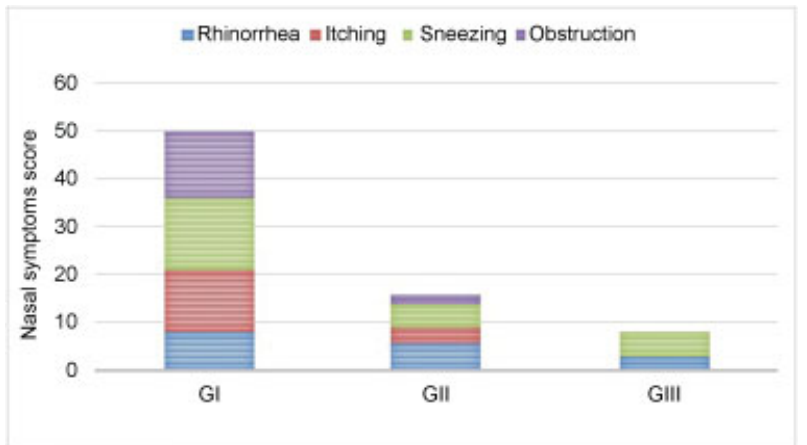

Fig. 1 Shows clinical improvement of nasal symptoms in AR patients in response to immunotherapy.

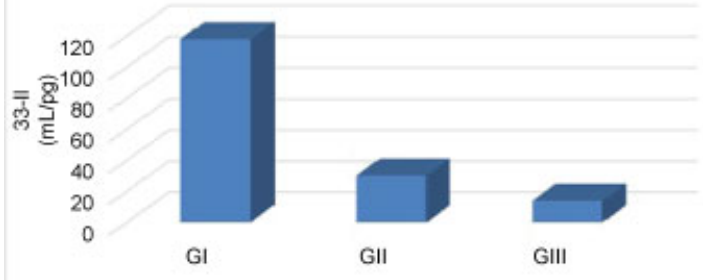

Fig. 2 Shows reduction in serum IL-33 levels in AR patients with immunotherapy.

\section{Discussion}

Allergic rhinitis is a worldwide health problem that affects patients from all racial groups, socioeconomic conditions, and ages. The incidence of positive skin test responses to an outdoor allergen (such as grass and tree pollen) among the US population has been approximated at $40 \%{ }^{7}$ Severe AR largely affects the health-related quality of life, work, and educational achievement, which causes a significant individual and economic problem. Allergic rhinitis and asthma are frequently comorbid disorders. Both considered as a part of the same allergic disease (united airway approach), as they affect the mucosa of the respiratory tract and shared by common underlying cellular processes. ${ }^{8}$

Pharmacotherapy offers symptomatic relief. But, some patients are unable to tolerate pharmacotherapy, and a substantial number report only partial or poor symptom control, particularly of systemic symptoms (for instance, fatigue or headache). ${ }^{9}$ Both subcutaneous progressively (SCIT) and sublingual immunotherapy (SLIT) are progressively being considered for this group of patients. In contrast to pharmacotherapy, the clinical benefits of SCIT and SLIT 
appear to be continued after termination of immunotherapy. ${ }^{10}$ There is evidence that IT can inhibit disease progression, development of new sensitizations, and onset of asthma together with treating symptoms. ${ }^{9}$

Intraleukin-33 induces $\mathrm{TH} 2$ cytokine production in $\mathrm{TH} 2$ cells, mast cells, basophils and eosinophils, suggesting that IL33 has the potential to induce $\mathrm{TH} 2$ cytokines-mediated allergic inflammation. ${ }^{11}$ Moreover, IL-33 in nasal secretions correlated significantly with the total nasal symptom score. ${ }^{3}$ Another study reported elevated level of IL-33 in sera of patients with AR allergic to tree and/or grass pollen and the correlation of IL-33 with the disease severity suggests that IL33 is involved in the pathogenesis of intermittent AR. ${ }^{4}$ Some studies had shown that clinical improvement is accompanied with a decrease in some immunological parameters after IT. ${ }^{12}$

The main aim of the study was to investigate the changes in clinical symptoms and serum IL-33 level before and after pollen IT in AR patients. We measured the levels of total IgE and IL-33 in the serum of patients with AR with a control group. Moreover, we evaluated the effects of a course of Phoenix dactylifera IT on the expression of these markers within the serum of patients with AR. We found higher levels of IgE in AR patients when compared with controls.

It is remarkable that serum levels of IL-33 were found significantly lower in the GII (Patients received IT for 6 months) and GIII (patients received IT for 2 years) AR patients than in GI (immunotherapy naïve) AR patients. This is in agreement with the study of Gluck et al. ${ }^{4}$ In this study, the investigators analyzed the serum level of IL-33 in patients with intermittent allergic rhinitis (IAR) sensitive to grass and/or tree pollen to evaluate if the serum level of IL-33 may be a parameter for the disease severity. The study found that serum levels of IL-33 in patients with IAR were comparable with patients with bronchial asthma and were significantly higher in patients with $\operatorname{IAR}(p=0.0035)$ and in patients with bronchial asthma $(p=0.008)$ than in controls. Serum levels of IL-33 correlated with disease severity. They suggested that IL-33 is included in the pathogenesis of IAR.

Other evidence came from the study of Kamekura. ${ }^{13}$ They investigated expression of IL-33 in the nasal epithelium of patients with AR and the mechanisms of the production of cytokines/chemokines induced by treatment with IL-33 using normal human nasal epithelial cells (HNECs) in vitro. Expression of IL-33 in normal and the AR, nasal mucosa was estimated by reverse transcription and real-time polymerase chain reactions and immunohistochemical methods. The serum levels of IL-33, IL-8 and GM-CSF were determined by ELISA. For in vitro experiments, HNECs in primary culture were used. The study showed a significant higher level of IL-33 in the sera of patients with AR than that in normal controls. The expression of IL-33 was significantly elevated in the epithelium of patients with AR. Another experimental report documented that ragweed pollen-driven endogenous IL-33 contributed to the development of AR responses. And concluded that IL-33 might present an important therapeutic target for the prevention of AR. ${ }^{14}$

However, controversial results were derived from the study of Asaka et al, ${ }^{3}$ who examined whether the levels of
IL-33 in sera and nasal secretions are unregulated in patients with AR, and they tested for correlations between the IL-33 level and the parameters of atopy and the nasal symptom score. The study included 24 Japanese cedar pollinosis patients (12 male and 12 female patients with a mean age of 47.7 years) with a history of moderate-to-severe AR, 14 house-dust-mite-sensitized patients with AR ( 9 male and 5 female patients with a mean age of 42 years) and 8 normal controls. Results declared that IL-33 protein was not detected in the serum of any of the subjects. However, the IL-33 level in nasal secretions was significantly elevated in patients with Japanese cedar pollinosis at peak season and in patients with perennial AR compared with Japanese cedar pollinosis patients at preseason and the normal controls. Furthermore, IL33 in nasal secretions correlated significantly with the total nasal symptom score.

In the present study, IT after 6 months and 2 years showed significant improvement of nasal symptoms when compared with treatment naïve patients and was associated with a decline in serum IL-33 levels. Our findings are in agreement with the Chinese study that discussed the expression of IL-33 and its receptor ST2 in the nasal mucosa in patients with AR. They concluded that the serum levels of IL-33 were significantly decreased after SIT treatment, suggesting that IL-33 may have a positive correlation with the severity of AR. ${ }^{15}$ Moreover, in murine studies, Haenuki et al elucidated that the induction of sneezing and eosinophilic and basophilic nasal inflammation in a ragweed pollen induced AR model was reduced in IL-33-deficient mice. Thus, these nasal allergic responses were mediated by endogenously released IL33. ${ }^{14}$ This ragweed pollen-driven IL-33 is essential for the development of sneezing and the nasal accumulation of eosinophils and basophils by increasing histamine release and inducing the production of chemoattractants from FceRI + mast cells and basophils, respectively. ${ }^{16}$ Furthermore, intranasal anti-IL-33 antibody significantly inhibited cigarette smoke-induced lung inflammation in mice, as evidenced by reducing levels of IL-33 and ST2, as well as decreased number of neutrophil and macrophage infiltration along with decreased expression of inflammatory cytokines (IL-1b, TNF, IL-17). ${ }^{17}$

The efficacy of IT in AR was recently approved by systematic review, concluding that updated meta-analyses confirmed statistically significant benefits for SCIT and SLIT for AR compared with placebo in adults and, to a lesser extent, in children. $^{18}$

Our study affords evidence that the serum level of IL-33 can be a sensitive marker that could associate with clinical improvement and play a role in the disease follow up. Further studies on large numbers of patients with longer duration of immunotherapy should be performed to better confirm these results and their clinical significance.

\section{Conclusion}

Interleukin-33 is elevated in treatment naïve AR patients (not subjected to immunotherapy). Its levels decline after IT, thus providing a way of assessing the clinical response to IT. 


\section{Conflict of Interest}

The authors report no conflict of interest.

\section{References}

1 Uzzaman A, Story R. Chapter 5: Allergic rhinitis. Allergy Asthma Proc 2012;33(Suppl 1):S15-S18

2 Cox L, Wallace D. Specific allergy immunotherapy for allergic rhinitis: subcutaneous and sublingual. Immunol Allergy Clin North Am 2011;31(03):561-599

3 Asaka D, Yoshikawa M, Nakayama T, Yoshimura T, Moriyama H, Otori N. Elevated levels of interleukin-33 in the nasal secretions of patients with allergic rhinitis. Int Arch Allergy Immunol 2012;158 (Suppl 1):47-50

4 Glück J, Rymarczyk B, Rogala B. Serum IL-33 but not ST2 level is elevated in intermittent allergic rhinitis and is a marker of the disease severity. Inflamm Res 2012;61(06):547-550

5 Schatz M, Meltzer EO, Nathan R, et al. Psychometric validation of the rhinitis control assessment test: a brief patient-completed instrument for evaluating rhinitis symptom control. Ann Allergy Asthma Immunol 2010;104(02):118-124

6 Passalacqua G, Durham SR; Global Allergy and Asthma European Network. Allergic rhinitis and its impact on asthma update: allergen immunotherapy. J Allergy Clin Immunol 2007;119(04):881-891

7 Bousquet J, Khaltaev N, Cruz AA, et al; World Health Organization; GA(2)LEN; AllerGen. Allergic Rhinitis and its Impact on Asthma (ARIA) 2008 update (in collaboration with the World Health Organization, GA(2)LEN and AllerGen). Allergy 2008;63(Suppl 86):8-160

8 Shaaban R, Zureik M, Soussan D, et al. Rhinitis and onset of asthma: a longitudinal population-based study. Lancet 2008; 372(9643):1049-1057
9 Walker SM, Durham SR, Till SJ, et al; British Society for Allergy and Clinical Immunology. Immunotherapy for allergic rhinitis. Clin Exp Allergy 2011;41(09):1177-1200

10 Durham SR, Emminger W, Kapp A, et al. SQ-standardized sublingual grass immunotherapy: confirmation of disease modification 2 years after 3 years of treatment in a randomized trial. J Allergy Clin Immunol 2012;129(03):717-725.e5

11 Smith DE. IL-33: a tissue derived cytokine pathway involved in allergic inflammation and asthma. Clin Exp Allergy 2010;40(02): 200-208

12 Boghdadi G, Marei A, Ali A, Lotfy G, Abdulfattah M, Sorour S. Immunological markers in allergic rhinitis patients treated with date palm immunotherapy. Inflamm Res 2012;61(07):719-724

13 Kamekura S. Expression of IL-33 in the nasal epithelium of patients with AR and production of cytokines/ chemokines. Clin Exp Allergy 2012;80:531-538

14 Haenuki Y, Matsushita K, Futatsugi-Yumikura S, et al. A critical role of IL-33 in experimental allergic rhinitis. J Allergy Clin Immunol 2012;130(01):184-94.e11

15 Du Y, Luo Y, Yang C, Liu J, Wan J, Wang K. [Discussion IL-33 and its receptor ST2 associated with the pathogenesis of allergic rhinitis]. Lin Chung Er Bi Yan Hou Tou Jing Wai Ke Za Zhi 2015;29(09):811-814

16 YoshimotoT, Matsushita K. Innate-type and acquired-type allergy regulated by IL-33. Allergol Int 2014;63(Suppl 1):3-11

17 Theoharides TC, Petra AI, Taracanova A, Panagiotidou S, Conti P. Targeting IL-33 in autoimmunity and inflammation. J Pharmacol Exp Ther 2015;354(01):24-31

18 Dretzke J, Meadows A, Novielli N, Huissoon A, Fry-Smith A, Meads C. Subcutaneous and sublingual immunotherapy for seasonal allergic rhinitis: a systematic review and indirect comparison. J Allergy Clin Immunol 2013;131(05):1361-1366 